\title{
Exploration and Practice on the Integrated Training of Supervisory Control System
}

\author{
Xiaolin Feng ${ }^{1,2}$, Haijie Mao ${ }^{1,2}$, Zhanming $\mathrm{Li}^{1,2}$, Hui Chen ${ }^{1,2}$ \\ 1. College of Electrical and Information Engineering Lanzhou University of Technology Lanzhou, China \\ 2. Key Laboratory of Gansu Advanced Control for Industrial Processes Lanzhou, China
}

\begin{abstract}
The integrated training of supervisory control system (abbreviated as ITSCS), whose main task focuses on using related domain knowledge flexible, is discussed carefully with the background of teaching practice. A schematic diagram illustrating the relationship between these parts is put forward and explained. Organizing various knowledge comprehensively, integrating SIMATIC SIM platform with training efficiently, applying practice project in society with students' learning in university as much as possible, are all traits of the training. The present limitations and authors' own considerations about improving the training continually are also discussed at the end.
\end{abstract}

Index Terms: integrated training of supervisory control system; schematic diagram; engineering practice ability

(C) 2012 Published by MECS Publisher. Selection and/or peer review under responsibility of the International Conference on E-Business System and Education Technology

\section{Introduction}

After experienced the courses of configuration software, principle and application of PLC, computer control technique, industry database technique, etc, integrated training of supervisory control system (abbreviated as ITSCS) is put forward to impart students with the capability of using what they have learned together. The training last about three weeks, divide all students into several groups with each has 6 or 7 to work on a specific supervisory control system. So how to organize ITSCS efficiently and improve the process with what the teaching facilities could rely on are main factors that the teachers should emphasize.

\section{Organizing ITSCS}

Schematic diagram as Fig. 1 was formed after a period time of discussing, and conducted the training. There are about seven main parts shown in the schematic diagram, that include database, develop tools of foreground software, MCU development tools, various data acquisition cards, commercial configuration software,

This work is supported by the National Professional-level Building Points (Grant No. TS12517), the Gansu Provincial Professional-level Building Points (Automation) and Teaching Research Project of Lanzhou Uni versity of Technology in China(Grant No. 201011).

* Corresponding author.

E-mail address: Splendor2003@126.com 
SIMATIC Step7, KEPServer and SIMATIC S7300 PLC. Although the students had learned some knowledge related with these parts before, it was still a challenging task for them to accomplish the task successfully within limited time. Students had learned configuration software before, but had only little knowledge about designing interface of system with VB or Delphi. Although they had the concept about AD, DA, DI and DO channels after learned MCU and PLC, they still had no the experiences of communicating with different data acquisition cards. KEPServer had been introduced and demonstrated in class before, but students still need to set up communications between various configuration software and KEPServer with their own hands. So for students, there are still a lot of new knowledge to learn.

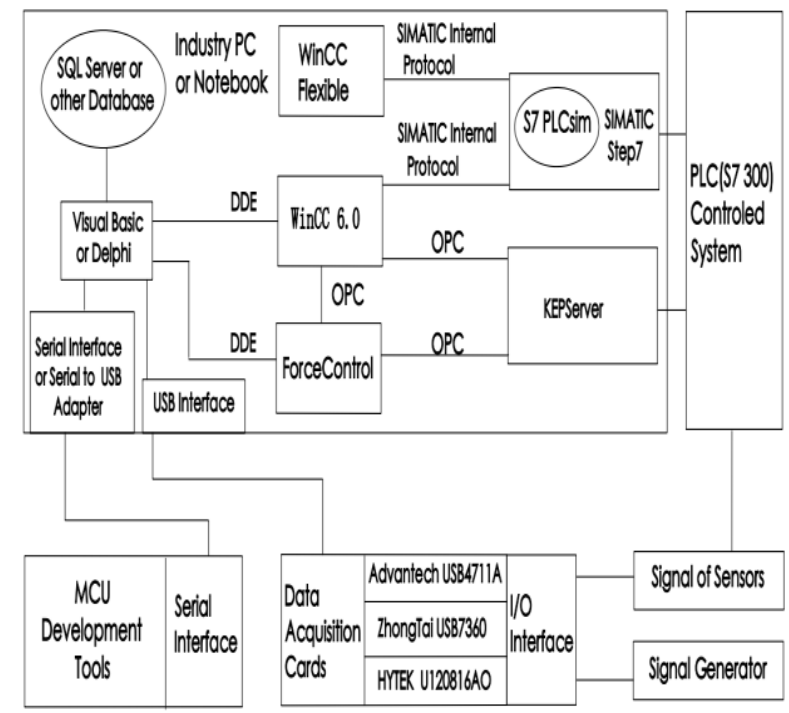

Figure 1. The schematic diagram of ITSCS

\section{A. Topics of Various Tasks}

Six topics aiming at different system was selected as below:

- Liquid level control system

- Five storey elevator control system

- Materials blend control system

- Design of dust-removal system

- Crossroad light control system

- Beer automatic packing system

\section{B. Accomplishing a Spicific Task}

For example of liquid level control system mentioned above, the control logic downloaded to SIMATIC S7 300 should be organized under Step7 before tested with PLCsim. WinCC Flexible and WinCC could get data of PLC directly, but training students to using simulation tools supported by Siemens automation platform should be an important point. At present, Siemens automation platform allow engineers to configure their project under WinCC or WinCC Flexible with help of PLCsim insider of Step7 without true panel or PLC ${ }^{[1-3]}$. Comparing with many other automation platforms whose simulation functions are limited, Siemens brings a convenient and practical tool to engineers and lets their job become easy ${ }^{[4]}$. So in ITSCS, mastering using simulation tools of Siemens to simply their work now should be emphasized. Fig. 2 shows how to simulate working way between S7-PLCsim and Wincc for liquid level of one tank in system (the system has at least two tank). 


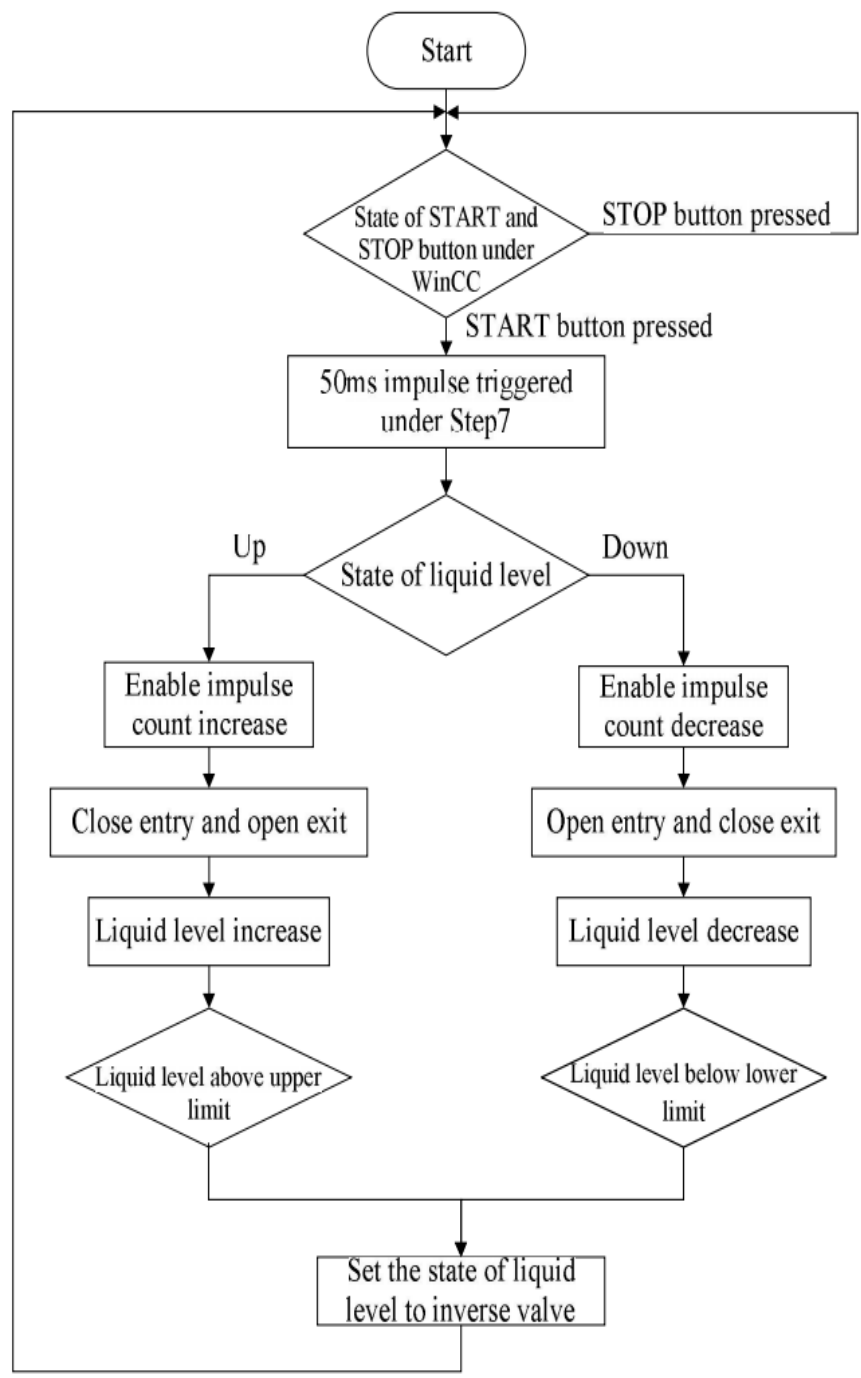

Figure 2. The flow-process diagram of controlling liquid value of a tank in the level control system

Fig. 3 shows the organized logic which controlled the level of tank in the form of LD and S7-PLCsim working window. When simulation begins, a START button insider WinCC picture would trigger flowing in LD. The real lines mean the logic could go through while the dashed lines mean the demanded conditions still could not be satisfied. Except S7-PLCsim working window could supply the function of start and stop the running of PLC, it could also let its users read or force the values of registers. It will bring more convenience in the process of debugging. WinCC Flexible and S7-PLCsim could also work in simulation way as WiCC and S7-PLCsim do. 


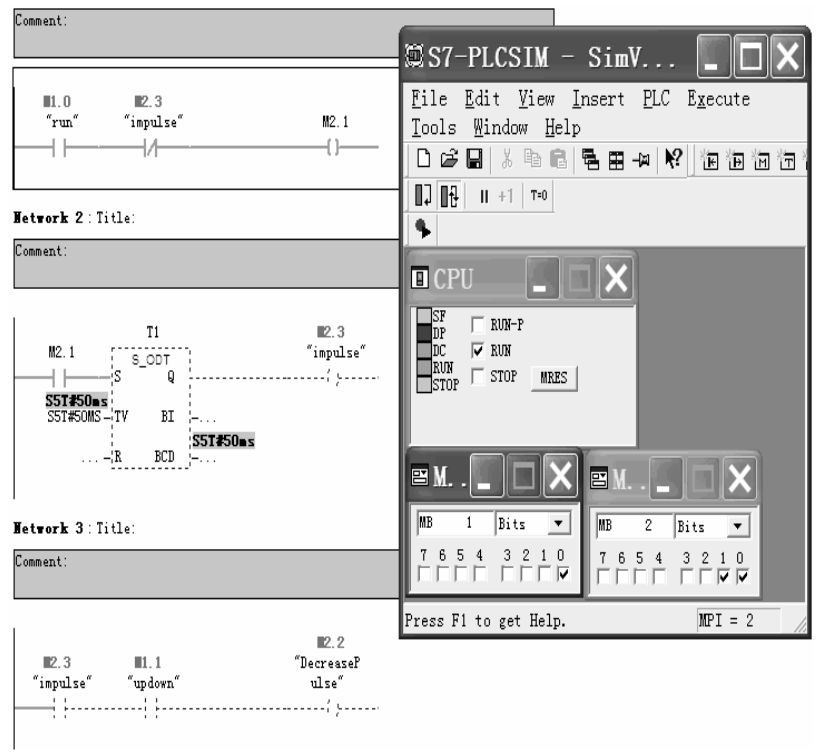

Figure 3. The captured vido showing simulation process insider Step7

In the curriculum of configuration software, WinCC and ForceControl had been introduced at same time. Now in ITSCS, communication between different configuration software is recommended because this always happen in practice industry factory. OPC is the most common protocol which supports communication between two configuration software ${ }^{[5-6]}$. Sometimes $\mathrm{I} / \mathrm{O}$ drivers embedded in configuration software could ensure its connection with controller on site smoothly, but not always happen ${ }^{[7]}$. So relying on third party such as KEPServer, then reading or writing these data with help of KEPServer become a proper way for configuration software. OPC is the only protocol that KEPServer carries. According to what mentioned above, understanding principles of OPC and mastering setting up communication between different software through OPC are all very important points for students.

VB and Delphi are all most commonly used develop tools as foreground software ${ }^{[7-9]}$. They are easy for students to learn rudiment and accomplish a simple task. Under VB or Delphi environment, students are demanded to collect temperature, humidity or other switch signal send from MCU development tools or different data acquisition cards. VB or Delphi could get other information through configuration software and stores all what they have got into database together with the steps of time or operator's commands ${ }^{[10]}$. Students was also demanded to supply the function that let operator to retrieve data with their selected conditions.

Based on what had learned about singlechip, MCU development tools integrating with various resources such as DS18b20 or SHT 10, was adopted in ITSCS to help students getting environment parameters of temperature and humidity. The supporting program organized under Keil should be downloads into MCU such as Atmel89s52 in advance, then these parameters would be transmitted to computer through serial port. Certainly if the notebook has not serial port, USB to serial port adapter is needed to add a virtual serial port in notebook.

Using ActiveX control as MScomm under VB, VCL controls as spcomm or TurboPower under Delphi, parameters uploaded by MCU would be collected and shown in pictures. Collected parameters representing the value of temperature and humidity could also be relayed to configuration software and shown in pictures of WinCC and ForceControl through DDE.

Sometimes on project site, measured signals come from data acquisition cards through USB interface. So three kinds of data acquisition cards such as Advantech 4711A, ZhongTai USB 7360, HYTEK U120816AO were provided to students. Help manuals was available and students could learn how to communicate with different data acquisition cards under environment of VB or Delphi. Usually the data acquisition cards carry multichannels supporting functions include DI, DO, AI and $\mathrm{AO}^{[11-13]}$. Some data acquisition cards even integrate 
programmable counter/timer and pulse output channel. So signal generator and oscillograph were provided and let students exercise working with such signals, although this has no direct relationship with their specific task.

\section{Strengthen Cultivation of Engineering Practice ability}

ITSCS should emphasize important of connect what students have learned from textbooks with practice projects in society. So before consider organizing the training, how to strengthening cultivation of engineering practice ability of students is the first factor. The teachers who conducted ITSCS had the experiences of participating with practice engineering projects. These projects included monitored control system of stage mechanical equipment in Qujing, Yunnan, P.R.China., 20Kg aluminium ingot casting machine control system exported to the Republic of Kazakhstan , copperplate automatic packaging line in Jinchuan, Gansuu, P.R.China. , etc. During the time of participating these projects, our teachers also considered how to impart these practice engineering experiences to students. Fig. 4 shows the configured picture under WinCC. In ITSCS the supervising system of $20 \mathrm{Kg}$ aluminium ingot casting machine hadn't been selected as direct design's object, but students could learn how to organize several picture reasonably in WinCC, how to set up alarm massages and let these massages shown in picture, how to draw trend picture of input analog channels, etc. Fig. 5 shows one picture configured for dust-removal monitored control system. The system is a practice engineering project which now selected as one task of ITSCS. When conducting students, new menu and layout of the system was encouraged to design while configured reference pictures such as Fig. 5 are available. At the same time, the system should also be configured under ForceControl and WinCC Flexible which had different configuration environments. Based on practice engineering project of copperplate automatic packaging line, VB, Delphi and database are also introduced to students and let them know how each of them playing an important role in industrial control field. Data acquisition card of Advantech PCI-1716, which controlled by VB or Delphi in industrial personal computer and used to communicate with PLC, was also introduced to students and thus broadened their horizon.

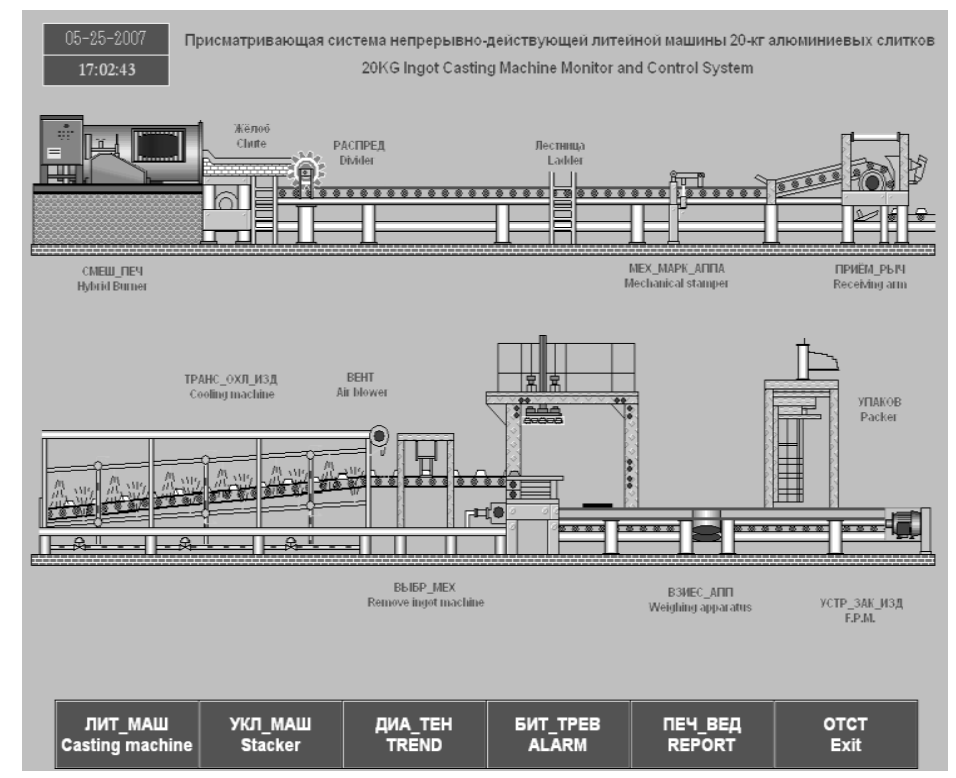

Figure 4. The configured picture of supervising system of $20 \mathrm{Kg}$ aluminium ingot casting machine 


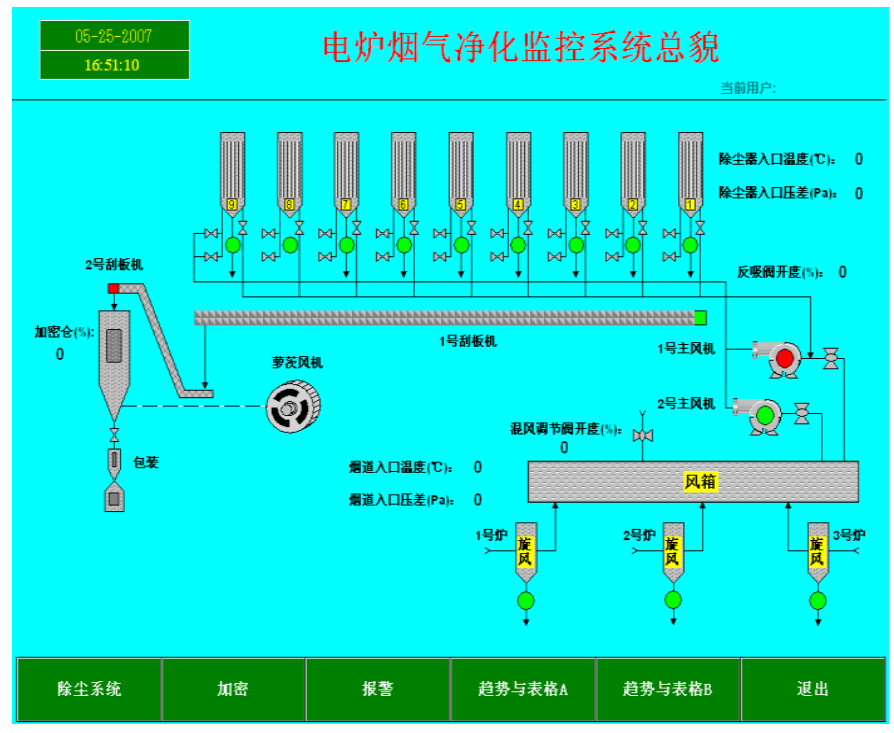

Figure 5. Configured picture of dust-removal monitored control system

ITSCS should emphasize that every students take part in the process and do every detail thing with their own hands. ITSCS should emphasize setting up of basic conception and learning to master communication ways between different software and hardware, although some of them are perhaps simple. So inoculating students with idea of important of basic and basic skill is what the teachers should do their best to do.

ITSCS should emphasize important of developing communication skills of students and let them learn how to exchange their own ideas in harmonious atmosphere. After all, ITSCS cover a wild range of knowledge and time is limited. Students shouldn't only care about their own job and limit their view within themselves. At the same time, teachers should let students know the important of sharing and encourage them to enjoy happiness of sharing. Based on one's own work, try to understand what other students in group have done and try to set up communication with other students' work. At last, each student could set up whole knowledge structure of ITSCS.

\section{Conclusion}

ITSCS constructs a huge network for students and try to integrate various import knowledge reasonably while emphasizing develop engineering practice ability. At present, about seventy students had accepted the training and admitted what the help ITSCS had brought to them. During the time of graduation design, students could do more based on what they had learned from ITSCS. Some students also found that after graduating, the knowledge that their work demanding had close relationship with ITSCS involving.

After all, there are still some points needed to be concluded and corrected. First of all, Labview should integrate with ITSCS legitimately and introduce to students. Labview is good at signal acquisition and processing which play an important role in many systems. Second point, at present the communication way between MCU and computer serial port is still simple. So setting up communication with help of opened protocol MB is worth doing and thus deepens the learning of conception of field bus for students. Third point, ITSCS should also encourage students try to setting up communication with different configuration software and data acquisition cards. In all, imparting students with various knowledge and engineering practice ability as much as possible is what ITSCS should emphasize and insist. 


\section{Acknowledgment}

Thanks are due to Professor Xinhua Yang, Wei Li, Associate Professor Weirong Liu and Senior Engineer Pinyuan Qiao for giving good proposal advice. The authors also get enlightenments in thinking ways and working methods from the work of the National Natural Science Foundation of China (Grant No.61005026) and the Gansu Provincial Science and Technology Planning of China (Grant No. 0916RJZA017).

\section{References}

[1] WinCC 6.0 reference manual [M]. Germany. Siemens Ltd., 2008.

[2] WinCC Flexible 2007 reference manual [M]. Germany. Siemens Ltd., 2007.

[3] S7-PLCSIM V5.3 [M]. Germany. Siemens Ltd., 2006

[4] Programming with STEP 7 [M]. Germany. Siemens Ltd., 2006

[5] ForceControl 6.0 reference manual(in Chinese) [M]. China. Beijing Sunway ForceControl Technology Co., Ltd, 2009.

[6] Huabo Liu. Application of configuration software of WinCC(in Chinese). [M]. China. China Machine Press, 2009.

[7] Jie Ren. Software engineering in practice(in Chinese) [M]. China. Tsinghua University Press, 2003.

[8] Dexin Meng. VB programming(in Chinese) [M]. China. Tsinghua University Press, 2008.

[9] Dinghua He. Delphi programming basic tutorial(in Chinese) [M]. China. Tsinghua University Press, 2008.

[10] Jian Liu. SQLserver database: text and cases(in Chinese) [M]. China. Tsinghua University Press, 2008.

[11] Advantech USB-4711A reference manual(in Chinese) [M]. China. Advantech Co., Ltd, 2006.

[12]Zhongtai USB7360 reference manual (in Chinese) [M]. China. Beijing Zhongtai Yanchuang Technology Co., Ltd, 2008.

[13]HYTEK U120816AO reference manual(in Chinese) [M]. China. HYTEK Automation Technology(Shanghai) Co., Ltd, 2008. 